\title{
COEFFICIENTS OF HARMONIC MAASS FORMS
}

\author{
KATHRIN BRINGMANN AND KEN ONO
}

\begin{abstract}
Harmonic Maass forms have recently been related to many different topics in number theory: Ramanujan's mock theta functions, Dyson's rank generating functions, Borcherds products, and central values and derivatives of quadratic twists of modular $L$-functions. Motivated by these connections, we obtain exact formulas for the coefficients of harmonic Maass forms of non-positive weight, and we obtain a conditional result for such forms of weight $1 / 2$. This extends earlier work of Rademacher and Zuckerman in the case of weakly holomorphic modular forms of negative weight.
\end{abstract}

\section{Introduction and Statement of Results}

In work which gave birth to the "circle method", Hardy and Ramanujan [19, 20] derived their famous asymptotic formula for the partition function

$$
p(n) \sim \frac{1}{4 n \sqrt{3}} \cdot e^{\pi \sqrt{2 n / 3}} .
$$

In celebrated work, Rademacher perfected [30] the method to derive the exact formula

$$
p(n)=2 \pi(24 n-1)^{-\frac{3}{4}} \sum_{k=1}^{\infty} \frac{A_{k}(n)}{k} \cdot I_{\frac{3}{2}}\left(\frac{\pi \sqrt{24 n-1}}{6 k}\right) .
$$

Here $I_{\ell}(x)$ is the $I$-Bessel function of order $\ell$, and $A_{k}(n)$ is the Kloosterman sum

$$
A_{k}(n):=\frac{1}{2} \sqrt{\frac{k}{12}} \sum_{\substack{x \\ x^{2} \equiv-24 n+1(\bmod 24 k) \\(\bmod 24 k)}} \chi_{12}(x) \cdot e\left(\frac{x}{12 k}\right),
$$

where $e(\alpha):=e^{2 \pi i \alpha}$ and $\chi_{12}(x):=\left(\frac{12}{x}\right)$.

These works make use of the fact that

$$
P(\tau)=\sum_{n=0}^{\infty} p(n) q^{n-\frac{1}{24}}=q^{-\frac{1}{24}} \prod_{n=1}^{\infty} \frac{1}{1-q^{n}},
$$

where $q:=e^{2 \pi i \tau}$, is a weight $-1 / 2$ weakly holomorphic modular form, a meromorphic modular form whose poles (if any) are supported at cusps. Rademacher and Zuckerman $[31,38,39]$ subsequently generalized (1.2) to obtain exact formulas for the coefficients of generic weakly holomorphic modular forms of negative weight.

The authors thank the support of the NSF, and the second author thanks the Manasse family and the Hilldale Foundation. This paper was written while the first author was in residence at the Max-Planck Institute. She thanks the institute for providing a stimulating environment. 
We recall that the partition generating function also has the Eulerian form

$$
\sum_{n=0}^{\infty} p(n) q^{n}=1+\sum_{n=1}^{\infty} \frac{q^{n^{2}}}{(1-q)^{2}\left(1-q^{2}\right)^{2} \cdots\left(1-q^{n}\right)^{2}} .
$$

By changing signs, one obtains Ramanujan's mock theta function

$$
f(q)=\sum_{n=0}^{\infty} a(n) q^{n}:=1+\sum_{n=1}^{\infty} \frac{q^{n^{2}}}{(1+q)^{2}\left(1+q^{2}\right)^{2} \cdots\left(1+q^{n}\right)^{2}} .
$$

The problem of obtaining an asymptotic formula for $a(n)$ is greatly complicated by the fact that $f(q)$ is not a modular form. In their doctoral theses (written under Rademacher), Andrews and Dragonette overcame this difficulty, and they confirmed a conjecture of Ramanujan by proving $[1,14]$ that

$$
a(n) \sim \frac{(-1)^{n-1}}{2 \sqrt{n-\frac{1}{24}}} \cdot e^{\pi \sqrt{\frac{n}{6}-\frac{1}{144}}}
$$

Andrews and Dragonette conjectured an exact formula for $a(n)$ to accompany (1.2). However, without a suitable description of the modular transformation properties of $f(q)$, this conjecture seemed out of reach. Then in his $2002 \mathrm{Ph} . \mathrm{D}$. thesis (written under Zagier), Zwegers [40, 41] provided this required theory. He related Ramanujan's mock theta functions to harmonic Maass forms. By combining his work with a lengthy argument, the authors proved the Andrews-Dragonette Conjecture [7].

Here we generalize this example to the general setting of harmonic Maass forms (see Section 2 for the definition), a class of automorphic forms which includes the weakly holomorphic modular forms. These results are of particular interest thanks to the recent appearance of harmonic Maass forms in a wide array of subjects: Ramanujan's mock theta functions $[2,7,8,9,10,37,40,41]$, Borcherds products $[3,4,11]$, derivatives and values of modular $L$-functions [13], probability theory [2, 6], and mathematical physics $[22,23,24,25,27,28,36]$.

Let $H_{2-k}(N, \chi)$ denote the space of weight $2-k$ harmonic Maass forms on $\Gamma_{0}(N)$ with Nebentypus character $\chi$, where we assume that $\frac{3}{2} \leq k \in \frac{1}{2} \mathbb{Z}$. The Fourier expansions of such forms are given in terms of the incomplete Gamma-function

$$
\Gamma(\alpha, x):=\int_{x}^{\infty} e^{-t} t^{\alpha-1} d t .
$$

More precisely, suppose that if $f(\tau) \in H_{2-k}(N, \chi)$. We then have that

$$
f(\tau)=\sum_{n \gg-\infty} c_{f}^{+}(n) q^{n}+\sum_{n<0} c_{f}^{-}(n) \Gamma(k-1,4 \pi|n| v) q^{n},
$$

where $\tau=u+i v \in \mathbb{H}$, with $u, v \in \mathbb{R}$. Obviously, each $f$ is the sum of two disjoint pieces, the holomorphic part of $f$

$$
f^{+}(\tau):=\sum_{n \gg-\infty} c_{f}^{+}(n) q^{n}
$$


and the nonholomorphic part of $f$

$$
f^{-}(\tau):=\sum_{n<0} c_{f}^{-}(n) \Gamma(k-1,4 \pi|n| v) q^{n} .
$$

Remark. Weakly holomorphic modular forms are those $f \in H_{2-k}(N, \chi)$ with $f^{-}=0$.

Generalizing earlier work of Rademacher and Zuckerman for weakly holomorphic modular forms of non-positive weight [30,31], and work of Bruinier and Hejhal [11, 21] for the harmonic Maass forms, we determine exact formulas for the coefficients $c_{f}^{+}(n)$. The idea is simple. We shall obtain our results by explicitly constructing Maass-Poincaré series which have poles supported (to arbitrary order) at individual cusps. We then relate a generic harmonic Maass form $f$ to that linear combination of Maass-Poincaré series which matches the divisor of $f$. If $2-k \leq 0$, then it turns out that $f$ equals this linear combination of Maass-Poincaré series.

We now define the functions which are required for these exact formulas. Throughout, we let $k \in \frac{1}{2} \mathbb{Z}$, and we let $\chi$ be a Dirichlet character modulo $N$, where $4 \mid N$ whenever $k \in \frac{1}{2} \mathbb{Z} \backslash \mathbb{Z}$. Using this character, for a matrix $M=\left(\begin{array}{ll}a & b \\ c & d\end{array}\right) \in \Gamma_{0}(N)$, we let

$$
\Psi_{k}(M):= \begin{cases}\chi(d) & \text { if } k \in \mathbb{Z} \\ \chi(d)\left(\frac{c}{d}\right) \epsilon_{d}^{2 k} & \text { if } k \in \frac{1}{2} \mathbb{Z} \backslash \mathbb{Z}\end{cases}
$$

where $\epsilon_{d}$ is defined by

$$
\epsilon_{d}:=\left\{\begin{array}{lll}
1 & \text { if } d \equiv 1 & (\bmod 4), \\
i & \text { if } d \equiv 3 & (\bmod 4),
\end{array}\right.
$$

and where $\left(\frac{c}{d}\right)$ denotes the extended Legendre symbol. In addition, if $T=\left(\begin{array}{ll}a & b \\ c & d\end{array}\right) \in \mathrm{SL}_{2}(\mathbb{Z})$, then we let

$$
\mu(T ; \tau):=(c \tau+d)^{2-k} .
$$

Moreover, for pairs of matrices $S, T \in \mathrm{SL}_{2}(\mathbb{Z})$, we let

$$
\sigma(T, S):=\frac{\mu(T ; S \tau) \mu(S ; \tau)}{\mu(T S ; \tau)} .
$$

Using this notation, we now define certain generic Kloosterman sums which are naturally associated with cusps of $\Gamma_{0}(N)$.

Suppose that $\rho=\frac{a_{\rho}}{c_{\rho}}=L^{-1} \infty,\left(L \in \mathrm{SL}_{2}(\mathbb{Z})\right)$ is a cusp of $\Gamma_{0}(N)$ with $c_{\rho} \mid N$ and $\operatorname{gcd}\left(a_{\rho}, N\right)=1$. Let $t_{\rho}$ and $\kappa_{\rho}$ be the cusp width and parameter of $\rho$ with respect to $\Gamma_{0}(N)$ (see 1.13). Suppose that $c>0$ with $c_{\rho} \mid c$ and $\frac{N}{c_{\rho}} \nmid c$. Then for integers $n$ and $m$ we have the Kloosterman sum

$$
K_{c}(2-k, \rho, \chi, m, n):=\sum_{\substack{0<d<c \\ 0<a<c t \\ a_{\rho} a \equiv-\frac{c}{c_{\rho}}\left(\bmod \frac{N}{c_{\rho}}\right) \\(a d, c)=1}} \frac{\sigma\left(L^{-1}, S\right)}{\Psi_{k}\left(L^{-1} S\right)} \cdot \exp \left(\frac{2 \pi i}{c}\left(\frac{\left(m+\kappa_{\rho}\right) a}{t_{\rho}}+n d\right)\right),
$$


where $S:=\left(\begin{array}{ll}a & b \\ c & d\end{array}\right) \in \mathrm{SL}_{2}(\mathbb{Z})$ is the unique matrix defined using the integers $a, c$, and $d$. Using properties of $\sigma$ and $\Psi_{k}$, one can easily show that (1.11) is well-defined. For similar Kloosterman sums we refer the reader to [32].

For convenience, we let $\mathcal{S}_{N}$ be a subset of $\mathrm{SL}_{2}(\mathbb{Z})$ with the property that $S_{1}^{-1} \infty$ and $S_{2}^{-1} \infty$ are inequivalent cusps in $\Gamma_{0}(N)$ whenever $S_{1}$ and $S_{2}$ are distinct elements of $\mathcal{S}_{N}$. For $M=\left(\begin{array}{ll}a & b \\ c & d\end{array}\right) \in \mathrm{SL}_{2}(\mathbb{Z})$, we define

$$
f_{M}(\tau):=(c \tau+d)^{k-2} f\left(\frac{a \tau+b}{c \tau+d}\right)
$$

where $\sqrt{\tau}$ is the principal branch of the holomorphic square root. Using this notation, we can speak of the Fourier expansion of a form $f$ at a cusp $\rho$. More precisely, if $L \in \mathcal{S}_{N}$ with $\rho=L^{-1} \infty$, then we have

$$
f_{\rho}(\tau)=\sum_{n \in \mathbb{Z}} a_{\rho}^{+}(n) q^{\frac{n+\kappa_{\rho}}{t_{\rho}}}+f_{\rho}^{-}(\tau) .
$$

We define the principal part of $f$ at $\rho$ by

$$
P_{f, \rho}(\tau):=\sum_{m+\kappa_{\rho}<0} a_{\rho}^{+}(m) q^{\frac{m+\kappa_{\rho}}{t_{\rho}}} .
$$

We shall use the principal parts of a form $f$ to determine our exact formulas. To this end, we identify, for each cusp $\rho$, its contribution to the exact formula. To make this precise, let $M=L^{-1}$ and $\mu=L \infty$. For positive $n$, we then define

$$
\begin{aligned}
& \mathcal{A}(N, 2-k, \chi, \rho, m, c ; n):= \\
& \quad-\frac{i^{k} 2 \pi}{t_{\mu}}\left|\frac{\left(-m+\kappa_{\mu}\right)}{t_{\mu} n}\right|^{\frac{k-1}{2}} \sum_{\substack{c>0 \\
c_{\mu} \mid c, \frac{N}{c_{\mu}} \nmid c}} \frac{K_{c}(2-k, \mu, \chi,-m,-n)}{c} \cdot I_{k-1}\left(\frac{4 \pi}{c} \sqrt{\frac{n\left|-m+\kappa_{\mu}\right|}{t_{\mu}}}\right) .
\end{aligned}
$$

Here $t_{\mu}$ and $\kappa_{\mu}$ are the cusp parameters for $\mu$ as in the notation above.

Using this notation, we define the order $\mathcal{N}$ Kloosterman approximation of $c_{f}^{+}(n)$ by

$$
\mathcal{C}(f, \mathcal{N} ; n):=\sum_{L \in \mathcal{S}_{N}} \sum_{m+\kappa_{\rho}<0} a_{\rho}^{+}(m) \sum_{c=1}^{\mathcal{N}} \mathcal{A}(N, 2-k, \chi, \rho, m, c ; n) .
$$

Moreover, we define $\mathcal{C}(f, \infty ; n)$ in the obvious way.

Remark. We stress again that $L$ and $\rho$ are related (throughout this section) by the formula $\rho=L^{-1} \infty$.

Theorem 1.1. If $f \in H_{2-k}(N, \chi)$ with $2 \leq k \in \frac{1}{2} \mathbb{Z}$, then for positive $n$ we have

$$
c_{f}^{+}(n)=\mathcal{C}(f, \infty ; n)
$$


Two remarks.

1) Using the asymptotic behavior of $I$-Bessel functions, an inspection of the principal parts of $f$ gives a minimal $\mathcal{N}$ for which

$$
\mathcal{C}(f, \mathcal{N} ; n) \sim c_{f}^{+}(n)
$$

Moreover, it is not difficult to show that

$$
c_{f}^{+}(n)=\mathcal{C}(f, \sqrt{n} ; n)+O_{f}\left(n^{\epsilon}\right) .
$$

2) Theorem 1.1 gives the results of Rademacher and Zuckerman in the very special case of those $f \in H_{2-k}(N, \chi)$ for which $2-k<0$ and $f^{-}=0$.

For weight $2-k=\frac{1}{2}$, we have a conditional result. To make it precise, we say that a form $f \in H_{\frac{1}{2}}(N, \chi)$ is good if the Maass-Poincaré series corresponding to nontrivial terms in the principal parts of $f$ are individually convergent.

Theorem 1.2. If $f \in H_{\frac{1}{2}}(N, \chi)$ is good, then there is a finite set $S_{\Theta}(f)$ of complex numbers such that for positive $n$ we have

$$
c_{f}^{+}(n)=\mathcal{C}(f, \infty ; n)+\mu(n)
$$

for some $\mu(n) \in S_{\Theta}(f)$. Moreover, if $n \neq d m^{2}$ for some $d \mid N$ and $m \in \mathbb{Z}^{+}$, then $\mu(n)=0$.

Four remarks.

1) Our method approximates each form as a linear combination of Poincaré series. In this paper, apart from the cases when the weight is $1 / 2$, this exactly determines the form. When the weight is $1 / 2$, this uniquely determines the form up to a holomorphic modular form, which must be a linear combination of theta functions. This contribution is given by $S_{\Theta}(f)$ and the numbers $\mu(n)$.

2) We believe that all $f \in H_{\frac{1}{2}}(N, \chi)$ are good. In earlier work we deduced convergence of such Maass-Poincaré series by making using of relationships between Kloosterman sums and Salié sums (see Section 4 of [7]), and by generalizing work of Goldfeld and Sarnak [18] on sums of Kloosterman sums (see [17]). It seems likely that a careful application of these ideas will prove that each such $f$ is indeed good.

3) Theorems 1.1 and 1.2 give exact formulas for harmonic weak Maass forms on congruence groups of the form $\Gamma_{1}(N)$. This follows from the fact that

$$
H_{2-k}\left(\Gamma_{1}(N)\right)=\oplus_{\chi} H_{2-k}(N, \chi),
$$

where the sum is over Dirichlet characters modulo $N$.

4) Apart from those harmonic Maass forms $f$ which are holomorphic modular forms (which in this paper can only happen if $2-k=1 / 2$ ), the results above imply that the $c_{f}^{+}(n)$ are not bounded by any power of $n$. There are arithmetic progressions of $n$ for which $c_{f}^{+}(n)$ grows subexponentially in $n$. We note that Bruinier and Funke [12] have 
a more general notion of a harmonic Maass form for which this claim is false. Indeed, Zagier's weight 3/2 Eisenstein series $g(\tau)$ [35], whose holomorphic part

$$
g^{+}(\tau)=-1 / 12+\sum_{n>0} h(-n) q^{n}
$$

is the generating function for Hurwitz class numbers, is such a form. Obviously, we have that $h(-n)=O\left(n^{\frac{1}{2}+\epsilon}\right)$ which does not have subexponential growth.

As mentioned earlier, harmonic Maass forms have appeared in a wide variety of contexts in recent years. We conclude the introduction with one application of these results.

In his work on Ramanujan's partition congruences

$$
\begin{aligned}
p(5 n+4) \equiv 0 & (\bmod 5), \\
p(7 n+5) \equiv 0 & (\bmod 7), \\
p(11 n+6) \equiv 0 & (\bmod 11) .
\end{aligned}
$$

Dyson defined [15] the rank of a partition to be its largest part minus its number of summands. If $0 \leq r<t$ are integers, then let $N(r, t ; n)$ denote the number of partitions with rank congruent to $r(\bmod t)$. In a recent paper [5], the first author obtained asymptotic formulas for each $N(r, t ; n)$ when $t$ is odd. Theorem 1.2 , combined with a generalization of Section 4 of [7], proves that these asymptotics can be extended to exact formulas.

Theorem 1.3. If $0 \leq r<t$, where $t$ is odd, then Theorem 1.2 gives an exact formula for $N(r, t ; n)$.

Remark. For brevity, we do not repeat the formulas from [5]. Theorem 1.2 proves that one obtains exact formulas, up to the coefficients of a linear combination of theta functions, by summing the first author's formulas to infinity.

This paper is organized as follows. In Section 2 we recall basic facts about harmonic Maass forms, and in Section 3 we construct the relevant Maass-Poincaré series. In Section 4 we prove Theorems 1.1 and 1.2.

\section{HARMONIC MAASS FORMS}

We recall the notion of a harmonic Maass form of weight $k \in \frac{1}{2} \mathbb{Z}$. Throughout let $\tau=u+i v \in \mathbb{H}$ with $u, v \in \mathbb{R}$, and we define the weight $k$ hyperbolic Laplacian

$$
\Delta_{k}:=-v^{2}\left(\frac{\partial^{2}}{\partial u^{2}}+\frac{\partial^{2}}{\partial v^{2}}\right)+i k v\left(\frac{\partial}{\partial u}+i \frac{\partial}{\partial v}\right) .
$$

For odd integers $d$, define $\epsilon_{d}$ by

$$
\epsilon_{d}:=\left\{\begin{array}{lll}
1 & \text { if } d \equiv 1 & (\bmod 4), \\
i & \text { if } d \equiv 3 & (\bmod 4) .
\end{array}\right.
$$


Definition 2.1. If $N$ is a positive integer (with $4 \mid N$ if $k \in \frac{1}{2} \mathbb{Z} \backslash \mathbb{Z}$ ) and $\chi$ is a Dirichlet character modulo $N$, then a weight $k$ harmonic Maass form on $\Gamma_{0}(N)$ with Nebentypus $\chi$ is any smooth function $M: \mathbb{H} \rightarrow \mathbb{C}$ satisfying the following:

(1) For all $A=\left(\begin{array}{ll}a & b \\ c & d\end{array}\right) \in \Gamma_{0}(N)$ and all $\tau \in \mathbb{H}$, we have

$$
M\left(\frac{a \tau+b}{c \tau+d}\right)= \begin{cases}\chi(d)(c \tau+d)^{k} M(\tau) & \text { if } k \in \mathbb{Z}, \\ \left(\frac{c}{d}\right)^{2 k} \epsilon_{d}^{-2 k} \chi(d)(c \tau+d)^{k} M(\tau) & \text { if } k \in \frac{1}{2} \mathbb{Z} \backslash \mathbb{Z} .\end{cases}
$$

(2) We have that $\Delta_{k} M=0$.

(3) There is a polynomial $P_{M}=\sum_{n \leq 0} c^{+}(n) q^{n} \in \mathbb{C}\left[q^{-1}\right]$ such that

$$
M(\tau)-P_{M}(\tau)=O\left(e^{-\epsilon v}\right)
$$

as $v \rightarrow+\infty$ for some $\epsilon>0$. Analogous conditions are required at all cusps.

Remark. We call $P_{M}(\tau)$ the principal part of $M$ at $\infty$, with analogous parts at others cusps.

Remark. Since holomorphic functions on $\mathbb{H}$ are harmonic, it follows that weakly holomorphic modular forms are harmonic Maass forms.

Harmonic Maass forms are related to classical modular forms thanks to the properties of differential operators. Here we require the differential operator

$$
\xi_{w}:=2 i v^{w} \cdot \frac{\bar{\partial}}{\partial \bar{\tau}}
$$

The following lemma ${ }^{1}$, which is a straightforward refinement of a proposition of Bruinier and Funke (see Proposition 3.2 of [12]), shall play a central role throughout this paper.

Lemma 2.2. If $f \in H_{2-k}(N, \chi)$, then

$$
\xi_{2-k}: H_{2-k}(N, \chi) \longrightarrow S_{k}(N, \bar{\chi})
$$

is a surjective map. Moreover, if

$$
f(\tau)=\sum_{n \gg-\infty} c_{f}^{+}(n) q^{n}+\sum_{n<0} c_{f}^{-}(n) \Gamma(k-1,4 \pi|n| v) q^{n},
$$

then we have that

$$
\xi_{2-k}(f)=-(4 \pi)^{k-1} \sum_{n=1}^{\infty} \overline{c_{f}^{-}(-n)} n^{k-1} q^{n} .
$$

We shall also require the following proposition.

Lemma 2.3. If $f \in H_{2-k}(N, \chi)$ has the property that $\xi_{2-k}(f) \neq 0$, then the principal part of $f$ is nonconstant for at least one cusp.

\footnotetext{
${ }^{1}$ The formula for $\xi_{2-k}(f)$ corrects a typographical error in [12].
} 
Proof. This lemma follows from the work of Bruinier and Funke [12]. Using their pairing $\{\bullet, \bullet\}$, one finds that $\left\{\xi_{2-k} f, f\right\} \neq 0$ thanks to its interpretation in terms of Petersson norms. On the other hand, Proposition 3.5 of [12] expresses this quantity in terms of the principal part of $f$ and the coefficients of the cusp form $\xi_{2-k}(f)$. An inspection of this formula reveals that at least one principal part of $f$ must be nonconstant.

\section{MAASS-POINCARÉ SERIES}

Here we use the method of Poincaré series to construct more general harmonic Maass forms with multiplier (i.e. generalizing the notion of Nebentypus). Such forms have been considered by Fay, Hejhal, and Niebur [16, 21, 29], and more recently by the authors and Bruinier [9, 11].

We closely follow the set-up in Rankin's classic text [32]. Suppose that $\Gamma$ is a subgroup of finite index in $\mathrm{SL}_{2}(\mathbb{Z})$ with $-I \in \Gamma, \frac{3}{2} \leq k \in \frac{1}{2} \mathbb{Z}$, and let $\nu(\bullet)$ be a multiplier system. Moreover, let $\rho:=L^{-1} \infty$ be a cusp and let $t$ and $\kappa$ be its cusp width and parameter for $\Gamma$. Let $\hat{\Gamma}_{\rho}$ be the stabilizer of $\rho$ in $\hat{\Gamma}$, the homogenization of $\Gamma$. For $T=\left(\begin{array}{ll}a & b \\ c & d\end{array}\right) \in \mathrm{SL}_{2}(\mathbb{Z})$, we let

$$
\mu(T ; \tau):=(c \tau+d)^{2-k}
$$

and for $T \in \Gamma$ we let

$$
\nu(T ; \tau):=\nu(T) \mu(T ; \tau) .
$$

For $s \in \mathbb{C}$ and $y \in \mathbb{R} \backslash\{0\}$, let

$$
\mathcal{M}_{s}(y):=|y|^{\frac{k}{2}-1} M_{\operatorname{sign}(y)(1-k / 2), s-\frac{1}{2}}(|y|),
$$

where $M_{\nu, \mu}(z)$ is the $M$-Whittaker function which is a solution to the differential equation

$$
\frac{\partial^{2} u}{\partial z^{2}}+\left(-\frac{1}{4}+\frac{\nu}{z}+\frac{\frac{1}{4}-\mu^{2}}{z^{2}}\right) u=0
$$

Using this function we let

$$
\phi_{s}(\tau):=\mathcal{M}_{s}(4 \pi v) e(u)
$$

where $\tau=u+i v$. If $m>0$, then we have the Maass-Poincaré series

$$
\mathcal{P}_{L}(\tau, m, \Gamma, 2-k, s, \nu):=\sum_{T \in \hat{\Gamma}_{\rho} \backslash \hat{\Gamma}} \frac{\phi_{s}\left(\frac{(-m+\kappa)}{t} L T \tau\right)}{\mu(L ; T \tau) \nu(T ; \tau)} .
$$

It is easy to check that $\phi_{s}(\tau)$ is an eigenfunction of $\Delta_{2-k}$ with eigenvalue

$$
s(1-s)+\frac{k^{2}-2 k}{4} \text {. }
$$

From this one can conclude, when the series converges absolutely, that $\mathcal{P}_{L}$ is an eigenfunction of $\Delta_{2-k}$. Next define for $S \in \mathrm{SL}_{2}(\mathbb{Z})$

$$
\Gamma^{S}:=S^{-1} \Gamma S
$$


and let $\nu^{S}$ be the multiplier defined on $\Gamma^{S}$ by

$$
\nu^{S}\left(S^{-1} T S\right):=\frac{\nu(T) \sigma(T, S)}{\sigma\left(S, S^{-1} T S\right)},
$$

where

$$
\sigma(T, S):=\frac{\mu(T ; S \tau) \mu(S ; \tau)}{\mu(T S ; \tau)} .
$$

We denote by $H_{2-k}(\Gamma, \nu)$ the space of harmonic Maass forms with multiplier $\nu$. This is the space of forms satisfying the conditions in Definition 2.1 where (2.3) is replaced, for all $S \in \Gamma$, by

$$
f(S \tau)=\frac{\mu(S ; \tau)}{\sigma(L, S)} f(\tau)
$$

The following lemma follows immediately from the properties described above for the functions in this construction.

Lemma 3.1. If $\operatorname{Re}(s)>1$, then the series in (3.4) is absolutely and uniformly convergent. Moreover, if $k>2$ and $s=k / 2$, then the series is in $H_{2-k}(\Gamma, \nu)$.

Now we return to the setting in the introduction, where we consider forms with Nebentypus $\chi$ on $\Gamma_{0}(N)$. Recalling (1.7), we set

$$
\mathcal{P}_{L}(\tau, m, N, 2-k, \chi):=\frac{1}{\Gamma(k)} \mathcal{P}_{L}\left(\tau, m, \Gamma_{0}(N), 2-k, \frac{k}{2}, \Psi_{k}\right) .
$$

We can determine the Fourier expansion of these Poincaré series at all cusps. The next theorem gives the holomorphic parts (1.5) for these series.

Theorem 3.2. If $2 \leq k \in \frac{1}{2} \mathbb{Z}$, then $\mathcal{P}_{L}(\tau, m, N, 2-k, \chi)$ is in $H_{2-k}(N, \chi)$. Moreover, the following are true:

(1) We have

$$
\mathcal{P}_{L}^{+}(\tau, m, N, 2-k, \chi)=\delta_{\infty, \rho} \cdot \frac{q^{-m}}{\Psi_{k}\left(L^{-1}\right) \sigma\left(L, L^{-1}\right)}+\sum_{n \geq 0} a^{+}(n) q^{n},
$$

where $\delta_{\infty, \rho}=0$, unless $\infty \sim \rho$ in $\Gamma_{0}(N)$, in which case it is 1 . Moreover, if $n>0$, then

$a^{+}(n)=-i^{k} 2 \pi\left|\frac{(-m+\kappa)}{t n}\right|^{\frac{k-1}{2}} \frac{1}{t} \sum_{\substack{c>0 \\ c_{\rho} \mid c, \frac{N}{c_{\rho}} \nmid c}} \frac{K_{c}(2-k, \rho, \chi,-m, n)}{c} \cdot I_{k-1}\left(\frac{4 \pi}{c} \sqrt{\frac{n|-m+\kappa|}{t}}\right)$.

(2) The principal part of $\mathcal{P}_{L}(\tau, m, N, 2-k, \chi)$ at the cusp $\mu=S \infty$ is given by

$$
\delta_{L, S} \cdot q^{\frac{(-m+\kappa \rho)}{t_{\rho}}}
$$

where $\delta_{L, S}=0$, unless $L=S$, in which case it equals 1 . 
Three remarks.

(1) Theorem 3.2 holds when $2-k=1 / 2$, provided that one can guarantee convergence in the formulas for the Fourier coefficients.

(2) Although Theorem 3.2 (1) is about the coefficients of the holomorphic parts of these Poincaré series, we also give the Fourier cefficients of the non-holomorphic parts in the proof of the theorem.

(3) Two features of Theorem 3.2 are important for us. Obviously, the exact formulas for the coefficients are important. Secondly, the fact that the principal parts are distinguished by cusps is vital. This fact allows us to piece together such Maass forms using the collection of principal parts at cusps.

Proof of Theorem 3.2. We first consider the more general Poincaré series from above and assume $\operatorname{Re}(s)>1$. We require more notation. Let $t_{1}$ (resp. $t_{2}$ ) be the cusp width of $\infty$ (resp. $\rho$ ) in $\Gamma$ and $\kappa_{1}\left(\right.$ resp. $\left.\kappa_{2}\right)$ the associated parameter. Define

$$
\mathcal{W}_{s}(y):=|y|^{\frac{k}{2}-1} W_{\left(1-\frac{k}{2}\right) \operatorname{sign}(y), s-\frac{1}{2}}(|y|),
$$

where $W_{\nu, \mu}(z)$ is the standard $W$-Whittaker function. Define the Kloosterman sum

$$
K_{c}(\rho, \Gamma, \nu, m, n):=\sum_{\left(\begin{array}{ll}
a & b \\
c & d
\end{array}\right)=S \in \mathcal{F}_{L}(c)} \frac{\sigma\left(L^{-1}, S\right)}{\nu\left(L^{-1} S\right)} \exp \left(\frac{2 \pi i}{c}\left(\frac{\left(m+\kappa_{2}\right) a}{t_{2}}+\frac{\left(n+\kappa_{1}\right) d}{t_{1}}\right)\right),
$$

where $\mathcal{F}_{L}(c)$ consists of all matrices $S \in L \Gamma$ for which

$$
0 \leq d<c t_{1} \quad 0 \leq a<c t_{2}
$$

One can compute the Fourier expansion of the Poincaré series using Poisson summation. Then the calculation boils down to computing integrals of the form

$$
\int_{\mathbb{R}} \tau^{2-k} \exp \left(-2 \pi i x(\kappa+n)-2 \pi i \lambda \operatorname{Re}\left(\frac{1}{\tau}\right)\right) \mathcal{M}_{s}\left(-4 \pi \lambda \operatorname{Im}\left(\frac{1}{\tau}\right)\right) d x
$$

This integral can be computed using pages 32-33 of [11]. This yields the following Fourier expansion:

$\mathcal{P}_{L}(\tau, m, \Gamma, 2-k, s, \nu)=\delta_{L, \Gamma} \cdot \frac{\mathcal{M}_{s}\left(\frac{4 \pi\left(-m+\kappa_{1}\right) y}{t_{1}}\right) e\left(\frac{\left(-m+\kappa_{1}\right)(x+r)}{t_{1}}\right)}{\nu\left(L^{-1} U^{r}\right) \sigma\left(L, L^{-1}\right)}+\sum_{n \in \mathbb{Z}} a_{y}(n) e\left(\frac{x}{t_{1}}\left(n+\kappa_{1}\right)\right)$,

where $\delta_{L, \Gamma}=0$ unless $L^{-1} U^{r} \in \Gamma$ for some $r \in \mathbb{Z}$ with $U:=\left(\begin{array}{ll}1 & 1 \\ 0 & 1\end{array}\right)$ in which case it is equal to 1 . In this case we have in particular that $t_{1}=t_{2}$ and $\kappa_{1}=\kappa_{2}$. Moreover the coefficients $a_{y}(n)$ are given as follows. 
(1) If $n+\kappa_{1}<0$, then

$$
\begin{aligned}
a_{y}(n)=-i^{k} \frac{2 \pi \Gamma(2 s)}{\Gamma\left(s+\frac{k}{2}-1\right)}\left|\frac{t_{1}\left(-m+\kappa_{2}\right)}{t_{2}\left(n+\kappa_{1}\right)}\right|^{\frac{k-1}{2}} \mathcal{W}_{s}\left(\frac{4 \pi\left(n+\kappa_{1}\right) y}{t_{1}}\right) \\
\frac{1}{t_{1}} \sum_{c>0} \frac{K_{c}(\rho, \Gamma, \nu,-m, n)}{c} \cdot J_{2 s-1}\left(\frac{4 \pi}{c} \sqrt{\frac{\left|-m+\kappa_{2}\right|\left|n+\kappa_{1}\right|}{t_{1} t_{2}}}\right),
\end{aligned}
$$

where $J_{\ell}$ is the Bessel function of order $\ell$.

(2) If $n+\kappa_{1}=0$, then

$$
\begin{array}{r}
a_{y}(n)=-i^{k} 2^{k} \pi^{\frac{k}{2}+s} y^{\frac{k}{2}-s} t_{1}^{-2} t_{2}^{-\frac{k}{2}+1-s} \frac{\Gamma(2 s)}{(2 s-1) \Gamma\left(s-\frac{k}{2}+1\right) \Gamma\left(s+\frac{k}{2}-1\right)}\left|-m+\kappa_{2}\right|^{\frac{k}{2}+s-1} \\
\sum_{c>0} \frac{K_{c}(\rho, \Gamma, \nu,-m, 0)}{c^{2 s+1}}
\end{array}
$$

(3) If $n+\kappa_{1}>0$, then

$$
\begin{aligned}
a_{y}(n)=-i^{k} \frac{2 \pi \Gamma(2 s)}{\Gamma\left(s-\frac{k}{2}+1\right)}\left|\frac{t_{1}\left(-m+\kappa_{2}\right)}{t_{2}\left(n+\kappa_{1}\right)}\right|^{\frac{k-1}{2}} \mathcal{W}_{s}\left(\frac{4 \pi\left(n+\kappa_{1}\right) y}{t_{1}}\right) \\
\frac{1}{t_{1}} \sum_{c>0} \frac{K_{c}(\rho, \Gamma, \nu,-m, n)}{c} \cdot I_{2 s-1}\left(\frac{4 \pi}{c} \sqrt{\frac{\left|-m+\kappa_{2}\right|\left|n+\kappa_{1}\right|}{t_{1} t_{2}}}\right) .
\end{aligned}
$$

Using special values of Whittaker functions, we obtain

$$
\begin{aligned}
& \mathcal{P}_{L}\left(\tau, m, \Gamma, 2-k, \frac{k}{2}, \nu\right)=\delta_{L, \Gamma} \cdot \frac{\Gamma(k) \cdot e\left(\frac{\left(-m+\kappa_{1}\right) r}{t_{1}}\right)}{\nu\left(L^{-1} U^{r}\right) \sigma\left(L, L^{-1}\right)} q^{\frac{\left(-m+\kappa_{1}\right)}{t_{1}}} \\
&\left(1-\frac{\Gamma\left(k-1, \frac{4 \pi\left(-m+\kappa_{1}\right) y}{t_{1}}\right)}{\Gamma(k-1)}\right)+\sum_{n+\kappa_{1} \geq 0} a(n) q^{\frac{n+\kappa_{1}}{t_{1}}} \\
&+\sum_{n+\kappa_{1}<0} a(n) \Gamma\left(k-1, \frac{4 \pi\left|n+k_{1}\right| y}{t_{1}}\right) q^{\frac{n+\kappa_{1}}{t_{1}}} .
\end{aligned}
$$

Here the coefficients $a(n)$ are given as follows. 
(1) If $n+\kappa_{1}<0$, then

$$
\begin{aligned}
a(n)=-i^{k} 2 \pi(k-1)\left|\frac{t_{1}\left(-m+\kappa_{2}\right)}{t_{2}\left(n+\kappa_{1}\right)}\right|^{\frac{k-1}{2}} \frac{1}{t_{1}} & \\
& \sum_{c>0} \frac{K_{c}(\rho, \Gamma, \nu,-m, n)}{c} \cdot J_{k-1}\left(\frac{4 \pi}{c} \sqrt{\frac{\left|-m+\kappa_{2}\right|\left|n+\kappa_{1}\right|}{t_{1} t_{2}}}\right) .
\end{aligned}
$$

(2) If $n+\kappa_{1}=0$, then

$$
a(n)=-i^{k}(2 \pi)^{k} t_{1}^{-2} t_{2}^{1-k}\left|-m+\kappa_{2}\right|^{k-1} \sum_{c>0} \frac{K_{c}(\rho, \Gamma, \nu,-m, 0)}{c^{k+1}} .
$$

(3) If $n+\kappa_{1}>0$, then

$$
\begin{gathered}
a(n)=-i^{k} 2 \pi \Gamma(k)\left|\frac{t_{1}\left(-m+\kappa_{2}\right)}{t_{2}\left(n+\kappa_{1}\right)}\right|^{\frac{k-1}{2}} \frac{1}{t_{1}} \\
\sum_{c>0} \frac{K_{c}(\rho, \Gamma, \nu,-m, n)}{c} \cdot I_{k-1}\left(\frac{4 \pi}{c} \sqrt{\frac{\left|-m+\kappa_{2}\right|\left|n+\kappa_{1}\right|}{t_{1} t_{2}}}\right) .
\end{gathered}
$$

The proof of Theorem 3.2 follows easily for $2-k \leq 0$. One merely observes that the defining series are convergent.

\section{Proof of Theorems 1.1 And 1.2}

Here we prove Theorems 1.1 and 1.2 simultaneously. Thanks to Theorem 3.2, we have an explicit linear combination of Maass-Poincaré series, say $\mathfrak{f} \in H_{2-k}(N, \chi)$, whose principal parts agree with the principal parts of $f$ up to additive constants. There are three possibilites:

Case 1. We have that $f-\mathfrak{f}$ is a holomorphic modular form. It can only be nonzero when $2-k=\frac{1}{2}$, in which case the Serre-Stark Basis Theorem implies that $f-\mathfrak{f}$ is a linear combination of theta functions. Either way, we obtain the relevant desired conclusions in Theorems 1.1 and 1.2.

Case 2. We have that $f-\mathfrak{f}$ is a weakly holomorphic modular form which is not a holomorphic modular form. Such a form must have a pole at a cusp. However, this cannot happen since we constructed $\mathfrak{f}$ so that the principal parts of $f-\mathfrak{f}$ are constant.

Case 3. We have that $f-\mathfrak{f}$ is a harmonic Maass form with a non-trivial nonholomorphic part. However, Lemma 2.3 shows that all such harmonic Maass forms have at least one principal part which is nonconstant. Therefore, this possibility never occurs.

This completes the proofs of the claimed exact formulas in Theorems 1.1 and 1.2. 


\section{REFERENCES}

[1] G. E. Andrews, On the theorems of Watson and Dragonette for Ramanujan's mock theta functions, Amer. J. Math. 88 No. 2 (1966), pages 454-490.

[2] G. E. Andrews, Partitions with short sequences and mock theta functions, Proc. Natl. Acad. Sci. USA 102 (2005), pages 4666-4671.

[3] R. E. Borcherds, Automorphic forms on $\mathrm{O}_{s+2,2}(\mathbb{R})$ and infinite products, Invent. Math. 120 (1995), pages 161-213.

[4] R. E. Borcherds, Automorphic forms with singularities on Grassmannians, Invent. Math. 132 (1998), pages 491-562.

[5] K. Bringmann, Asymptotics for rank partition functions, Trans. Amer. Math. Soc., accepted for publication.

[6] K. Bringmann and K. Mahlburg, Asymptotics for partitions without sequences, in preparation.

[7] K. Bringmann and K. Ono, The $f(q)$ mock theta function conjecture and partition ranks, Invent. Math. 165 (2006), pages 243-266.

[8] K. Bringmann and K. Ono, Dyson's ranks and Maass forms, Ann. of Math., accepted for publication.

[9] K. Bringmann and K. Ono, Lifting cusp forms to Maass forms with an application to partitions, Proc. Natl. Acad. Sci., USA 104, No. 10 (2007), pages 3725-3731.

[10] K. Bringmann, K. Ono, and R. Rhoades, Eulerian series as modular forms, J. Amer. Math. Soc., 21 (2008), pages 1085-1104.

[11] J. H. Bruinier, Borcherds products on $\mathrm{O}(2, l)$ and Chern classes of Heegner divisors, Springer Lect. Notes Math. 1780, Springer-Verlag (2002).

[12] J. H. Bruinier and J. Funke, On Two Geometric Theta Lifts, Duke Math. Journal 125 (2004), pages $45-90$.

[13] J. H. Bruinier and K. Ono, Heegner divisors, L-functions and harmonic weak Maass forms, Ann. of Math., accepted for publication.

[14] L. Dragonette, Some asymptotic formulae for the mock theta series of Ramanujan, Trans. Amer. Math. Soc. 72 No. 3 (1952), pages 474-500.

[15] F. Dyson, Some guesses in the theory of partitions, Eureka (Cambridge) 8 (1944), pages 10-15.

[16] J. D. Fay, Fourier coefficients of the resolvent for a Fuchsian group, J. Reine Angew. Math. 293/294 (1977), pages 143-203.

[17] A. Folsom and K. Ono, Duality involving the mock theta function $f(q)$, J. London Math. Soc. 77 (2008), pages 320-334.

[18] D. Goldfeld and P. Sarnak, Sums of Kloosterman sums, Invent. Math. 71 (1983), pages 243-250.

[19] G. H. Hardy and S. Ramanujan, Une formule asymptotique pour le nombre des partitions de $n$ [Comptes Rendus, 2 Jan. 1917] (French) [An asymptotic formula for the number of partitions of $n$ ] Collected papers of Srinivasa Ramanujan, pages 239-241, AMS Chelsea Publ., Providence, RI, 2000.

[20] G. H. Hardy and S. Ramanujan, Asymptotic formulae in combinatory analysis [Proc. London Math. Soc. (2) 16 (1917), Records for 1 March 1917]. Collected papers of Srinivasa Ramanujan, 244, AMS Chelsea Publ., Providence, RI, 2000.

[21] D. A. Hejhal, The Selberg trace formula for PSL $(2, R)$. Vol. 2, Springer Lect. Notes in Math. 1001 Springer-Verlag, Berlin, 1983.

[22] K. Hikami, Mock (false) theta functions as quantum invariants, Regul. Chaotic Dyn. 10 (2005), pages $163-174$.

[23] K. Hikami, Transformation formula of the "second" order mock theta function, Lett. Math. Phys. 75 (2006), pages 93-98.Maa 
[24] K. Hikami, Hecke type formula for the unified Witten-Reshetikhin invariants as higher order mock theta functions, Int. Math. Res. Not. 7 (2007), 32 pp.

[25] R. Lawrence and D. Zagier, Modular forms and quantum invariants of 3-manifolds, Asian J. Math. 3 (1999), pages 93-107.

[26] J. Lehner, A partition function connected with the modulus five. Duke Math. J. 8, (1941), pages 631-655.

[27] A. Malmendier and K. Ono, SO(3) Donaldson invariants of $C P^{2}$ and mock theta functions, preprint.

[28] A. Mellit and S. Okada, Joyce invariants for K3 surfaces and mock theta functions, preprint.

[29] D. Niebur, A class of nonanalytic automorphic functions, Nagoya Math. J. 52 (1973), pages $133-145$.

[30] H. Rademacher, On the expansion of the partition function in a series, Ann. of Math. (2) 44, (1943), pages 416-422.

[31] H. Rademacher and H. S. Zuckerman, On the Fourier coefficients of certain modular forms of positive dimension, Ann. of Math. (2) 39 (1938), no. 2, pages 433-462.

[32] R. Rankin, Modular forms and functions, Cambridge University Press, Cambridge-New YorkMelbourne, 1977.

[33] J.-P. Serre and H. Stark, Modular forms of weight 1/2, Springer Lect. Notes 627, Springer-Verlag, Berlin, (1977), pages 27-67.

[34] M. Wakimoto, Representation theory of affine superalgebras at the critical level, Proc. Int. Congress of Math., Vol. II, (Berlin, 1998), Doc. Math. 1998, Extra Vol., II, pages 605-614.

[35] D. Zagier, Nombres de classes et formes modulaire de poids 3/2, C.R. Acad. Sci. Paris 281, 1975, A883-A886.

[36] D. Zagier, Vassiliev invariants and a strange identity related to the Dedekind eta-function, Topology 40 (2001), pages 945-960.

[37] D. Zagier, Ramanujan's mock theta functions and their applications [d'après Zwegers and Bringmann-Ono], Séminaire Bourbaki, 60éme année, 2006-2007, no,. 986.

[38] H. S. Zuckerman, On the coefficients of certain modular forms belonging to subgroups of the modular group, Trans. Amer. Math. Soc., 45 (1939), pages 298-321.

[39] H. S. Zuckerman, On the expansions of certain modular forms of positive dimension, Amer. J. Math. 62 (1940), pages 127-152.

[40] S. P. Zwegers, Mock $\vartheta$-functions and real analytic modular forms, $q$-series with applications to combinatorics, number theory, and physics (Ed. B. C. Berndt and K. Ono), Contemp. Math. 291, Amer. Math. Soc., (2001), pages 269-277.

[41] S. P. Zwegers, Mock theta functions, Ph.D. Thesis, Universiteit Utrecht, 2002.

Mathematisches Institut, Universität Köln, Weyertal 86-90, D-50931 Köln, Germany

E-mail address: kbringma@math.uni-koeln.de

Department of Mathematics, University of Wisconsin, Madison, Wisconsin 53706

E-mail address: ono@math.wisc.edu 